\title{
Where do flare ribbons stop?
}

\author{
CHEN PengFei ${ }^{1,2^{*}}$, SU JiangTao ${ }^{2}$, GUO Yang ${ }^{1} \&$ DENG YuanYong ${ }^{2}$ \\ ${ }^{1}$ Department of Astronomy, Nanjing University, Nanjing 210093, China; \\ ${ }^{2}$ Key Laboratory of Solar Physics, National Astronomical Observatories, Chinese Academy of Sciences, Beijing 100012, China
}

Received August 31, 2011; accepted September 21, 2011; published online November 9, 2011

The standard flare model, which was proposed based on observations and magnetohydrodynamic theory, can successfully explain many observational features of solar flares. However, this model is just a framework, with many details awaiting to be filled in, including how reconnection is triggered. In this paper, we address an unanswered question: where do flare ribbons stop? With the data analysis of the 2003 May 29 flare event, we tentatively confirmed our conjecture that flare ribbons finally stop at the intersection of separatrices (or quasi-separatrix layer in a general case) with the solar surface. Once verified, such a conjecture can be used to predict the final size and even the lifetime of solar flares.

solar flares, magnetic field, separatrix

Citation: Chen P F, Su J T, Guo Y, et al. Where do flare ribbons stop? Chin Sci Bull, 2012, 57: 1393-1396, doi: 10.1007/s11434-011-4829-9

Owing to the omnipresence of magnetic field in the solar atmosphere, the Sun presents a variety of activities, which are modulated with the 11-year solar cycles. One of the spectacular phenomena is solar flares. They represent the typical process that magnetic energy accumulated gradually in the corona is converted rapidly into thermal and kinetic energies. Morphologically, flares are classified into two types, i.e. compact and two-ribbon flares (e.g. [1]). Compact flares are characterized by a compact flare loop, which does not show significant change in shape, whereas two-ribbon flares are characterized by flaring loop expansion and bright ribbon separation. Two-ribbon flares attracted more attention since they are frequently related to coronal mass ejections (CMEs).

To explain the appearance of the flaring loops, two ribbons, and their association with filament eruptions, a standard flare model was gradually developed by Carmichael [2], Sturrock [3], Hirayama [4], and Kopp \& Pneuman [5], which was later called CSHKP model. The standard flare model, where magnetic reconnection below an erupting flux rope is the key ingredient, was supported by a lot of observations, such as the discoveries of chromospheric evaporation, the cusp-shaped structure, the reconnection downflow, and inflow (see [6] for

*Corresponding author (email: chenpf@nju.edu.cn) a review). However, it should be kept in mind that such a model is just a framework, and many detailed processes inside it await to be clarified and understood theoretically; for example, how the reconnection is triggered in the highlyconducting plasma. Another unclarified issue is related to the flare ribbon separation.

The typical feature of two-ribbon flares in $\mathrm{H} \alpha$ or any other chromospheric wavelength is the ribbon separation. The separating speed reaches up to $50 \mathrm{~km} \mathrm{~s}^{-1}$ in the impulsive phase and decreases to $\leqslant 1 \mathrm{~km} \mathrm{~s}^{-1}$ in the decay phase [7]. One important question remaining unanswered is: where do the two ribbons finally stop? This paper is aimed to address such an issue.

\section{Our conjecture}

In the standard flare model, as a filament (or a magnetic flux rope in a general sense) erupts, the overlying field lines are stretched up, leading to the formation of a current sheet below the flux rope. As reconnection is triggered and goes on in the current sheet, the reconnected field lines below the reconnection area, along with the heated plasmas, pile up. The previously-heated loops cool down due to radiation and heat 
conduction. Accelerated around the reconnection area, energetic particles, along with thermal conduction, are transferred down along the separatrix or quasi-separatrix layer (QSL) to heat the chromosphere, forming $\mathrm{H} \alpha$ ribbons and plasma evaporation. These processes result in the typical observed features of two-ribbon flares, i.e. the apparent expansion of the flaring loop and the separation motion of the flare ribbons (e.g. [8]). Such processes can keep going if the magnetic field lines straddling over the flux rope extend to a long distance in the horizontal direction, i.e. in the case of a large-scale bipolar field. However, at least two factors may terminate such an on-going reconnection process. One is that the reconnected field lines pile up to reach the reconnection area, which then hinders the anti-parallel field lines from further reconnecting. This factor can account for compact flares as demonstrated by Chen et al. [9], but not for two-ribbon flares, where the current sheet extends up well above the flare loop. The second factor, which we propose to account for the limited lifetime of two-ribbon flares, is the existence of outer magnetic separatrices or QSLs.

The idea is explained in Figure 1, the central part of which is essentially the same as the CSHKP model, i.e. a flux rope with a null point below resides inside a filament channel. An inner magnetic separatrix (green dashed line) runs across the X-type null point and maps to the flare ribbons. Note that in 3-dimensions the null point is generalized to a quasi-separator (or hyperbolic flux tube, [10]) and the inner separatrices are QSLs (which include separatrices as a special case) [11]. The difference of Figure 1 from the classical CSHKP model is that there exist two outer magnetic separatrix (or QSL in 3-dimensions) segments on the two sides of the filament channel respectively, as indicated by the blue dashed lines above $S_{\text {out }}^{\mathrm{L}}$ and $S_{\text {out }}^{\mathrm{R}}$. Outside $S_{\text {out }}^{\mathrm{L}}-S_{\text {out }}^{\mathrm{R}}$ the field lines belong to different flux systems, which can either be open field (i.e. a coronal hole) or closed field. As the flux rope erupts, only the field lines straddling over the flux rope between $S_{\text {out }}^{\mathrm{L}}$ and $S_{\text {out }}^{\mathrm{R}}$ can be stretched up and experience reconnection below the flux rope. This means that the moving flare ribbons will finally stop at the intersections of the outer magnetic separatrices (or QSLs) with the solar surface, i.e. at $S_{\text {out }}^{\mathrm{L}}$ and $S_{\text {out }}^{\mathrm{R}}$.

To confirm such a conjecture, we analyze the 2003 May 29 flare event, and study the spatial relation between QSLs and the final positions of the flare ribbons.

\section{Observations and data analysis}

On 2003 May 29, a GOES X1.2-class flare occurred at S06W37 in the active region AR10365. The flare started at $\sim 00: 51$ UT and peaked at 01:05 UT. UV ribbons were almost invisible after $\sim 02: 00$ UT. It was a typical long-duration event, showing two ribbons separating gradually. The flare loops and ribbons were well observed by the Transition Region and Coronal Explorer (TRACE, [12]) with a high spatial resolution of $1^{\prime \prime}$ and a cadence of $\sim 3$ min. The photospheric vector magnetograms across the flare were obtained in Huairou Solar Observing Station (HSOS, [13]) with a pixel size of $0.35^{\prime \prime}$ and a cadence of $\sim 10 \mathrm{~min}$. The coalignment of the two datasets is accomplished with the help of the magnetogram Michelson Doppler Imager (MDI) aboard the Solar and Heliospheric Observatory (SOHO).

Figure 2 depicts the evolution of the flare in TRACE 1600 $\AA$, where two ribbons were observed to separate slowly. Ribbon 1 is separate from any other brightenings, whereas ribbon 2 , marked by an ellipse, is connected to a bright patch in the north. At 01:56:15 UT, the flare ribbons nearly approached their final positions before fading away. Note that the bright patch to the north of ribbon 2 persisted during the flare, whose nature is beyond the scope of this paper.

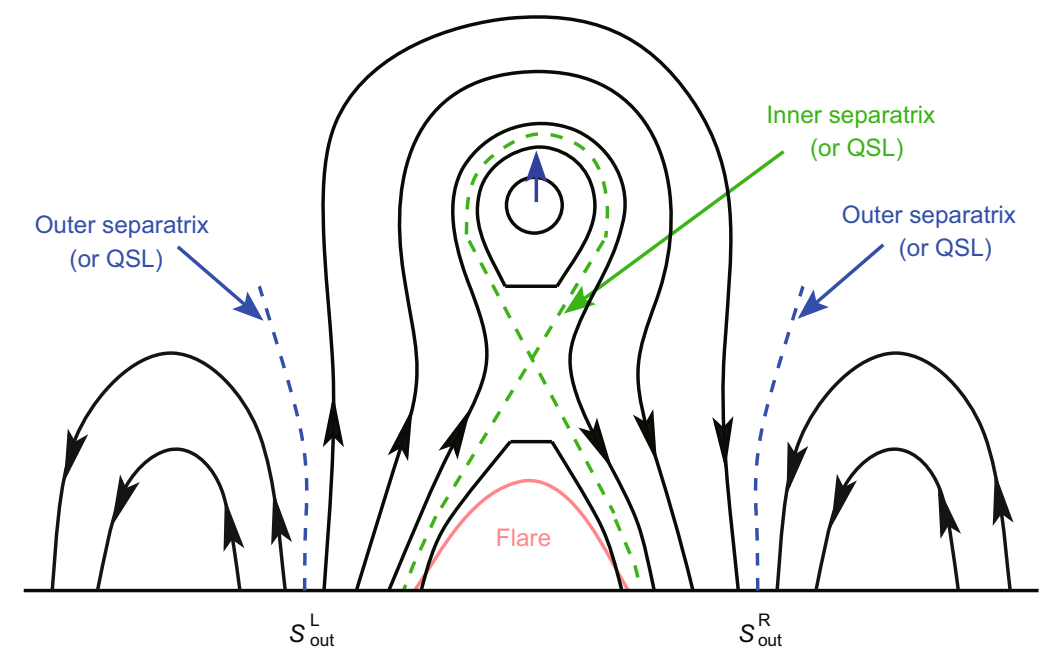

Figure 1 (Color online) A sketch of magnetic reconnection model with both inner and outer separatrices (or quasi-separatrix layers, QSLs) being considered, where the black solid lines are magnetic field lines. It is proposed in this paper that the flare ribbons would finally stop at the intersection of the outer separatrices (or QSLs) with the solar surface, i.e. $S_{\text {out }}^{\mathrm{L}}$ and $S_{\text {out }}^{\mathrm{R}}$. 

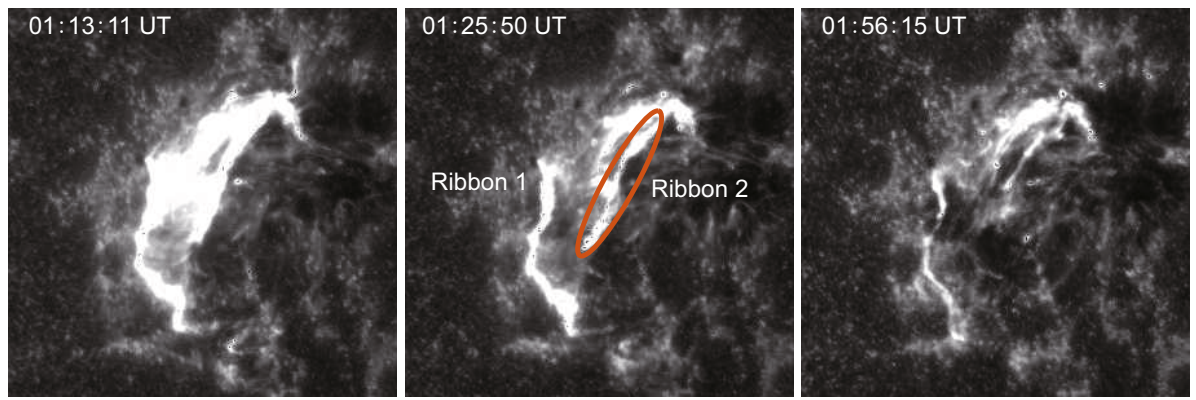

Figure 2 (Color online) Evolution of the 2003 May 29 solar flare in the TRACE 1600 Å wavelength showing the separation of two ribbons. North is up. The ribbons 1 and 2 are marked, and it is noted a bright patch to the north of ribbon 2 persisted during the flare.

The coronal magnetic field is extrapolated from the HSOS vector magnetogram before the flare peak, at 00:59 UT, with the non-linear force-free model [14]. Figure 3 shows the extrapolated coronal magnetic field lines with the photospheric magnetogram being rendered at the bottom. The different magnetic flux systems are clearly identified, and the boundaries between neighboring flux systems correspond to QSLs, across which field lines go divergently. The flaring ribbons at 01:56:15 UT (white lines) are located near the boundaries.

In order to compare the locations of the QSLs with those of the two ribbons more quantitatively, we calculate the squashing degree $Q$, which characterizes the magnetic connectivity, with $Q>>2$ corresponding to a QSL [15]. $Q$ is infinite at separatrices in theory, and is a very large value due to finite size of the numerical grid. The $Q$-map (yellow lines) is superimposed over the TRACE $1600 \AA$ intensity map at 01:56:15 UT in Figure 4. It can be seen that ribbon 2 near the end of the flare is almost exactly cospatial with the intersection of the QSL at the solar surface. Although ribbon 1 is also roughly cospatial with the intersection of the QSL, it is inclined with the QSL intersection with an angle of $20^{\circ}$. Note that no null point or bald patch exists in the modeled box, we are not sure whether the QSLs are separatrices.

\section{Discussion}

Magnetic QSLs play an important role in active region heating [16] and magnetic reconnection as well [17, 18]. Solar flares, either compact or two-ribbon flares, are widely explained in terms of magnetic reconnection [19]. In the reconnection model, magnetic QSLs (or separatrices as a special case) divide the reconnected field lines from the prereconnection field lines. Energetic particles and heat conduction are transported down to evaporate the chromospheric plasma to form flare loops. As the reconnection goes on, larger flaring loops are formed on top of previous ones, with the two footpoints (two ribbons in 3-dimensions) separating continually. According to such a reconnection model, at any time during a flare, the flare ribbons, i.e. the footpoints of the flare loops, are located at the intersection of this inner magnetic separatrix (or QSL) with the solar surface, as illustrated by Figure 1. As the reconnection proceeds, this inner separa-

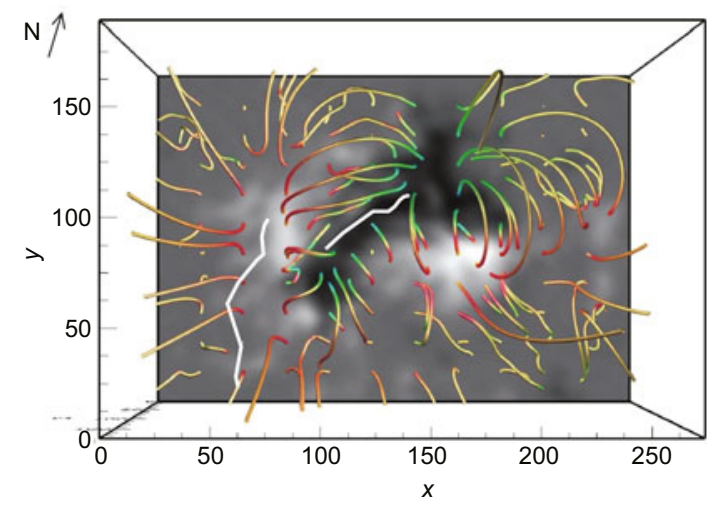

Figure 3 (Color online) Top view of the extrapolated coronal magnetic field (solid lines) that is anchored to the photospheric magnetograms (grayscale). The two white thick lines mark the locations of the two flare ribbons. North is indicated by the arrow at the top-left corner.

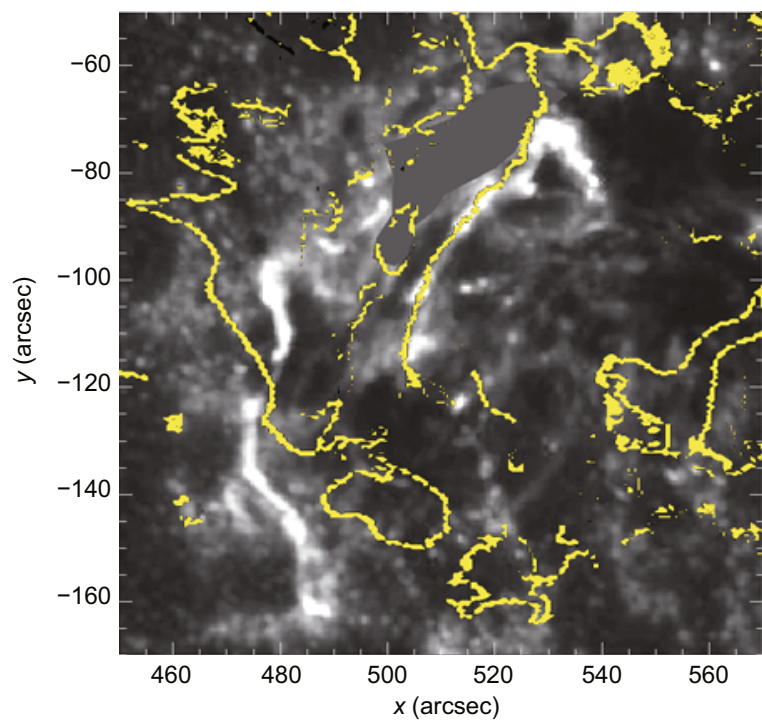

Figure 4 (Color online) Comparison between the final locations of the flare ribbons at 01:56:15 UT (gray-scale) with the $Q$-map (yellow lines corresponding to $Q=3100$ ) at 00:59 UT, which indicates that the final location of ribbon 2 is cospatial with the footpoints of a magnetic separatrix and that of ribbon 1 is roughly cospatial with the footpoints of separatrix. North is up. Note that the bright patch to the north of ribbon 2 is removed.

trix (or QSL) moves outward horizontally along with the flare ribbons. With this paper, we point out that there should also exist outer separatrices (or QSLs) which border the filament 
channel, as marked by $S_{\text {out }}^{\mathrm{L}}$ and $S_{\text {out }}^{\mathrm{R}}$ in Figure 1 . After all the field lines between the inner separatrices (or QSLs) and the outer separatrices (or QSLs) have reconnected, no more field lines are available for further reconnection, and magnetic reconnection is expected to halt. When this happens, the flare ribbons reach the outer separatrices (or QSLs). In this paper, we analyzed the 2003 May 29 flare event, and found that the final location of ribbon 2 well matches the outer QSL that is derived from the pre-flare magnetic field. Ribbon 1, however, only roughly matches the outer QSL. The probable reason for the slight discrepancy of ribbon 1 is that the field of view of the HSOS vector magnetogram is too small and ribbon 1 is very close to the edge of the field of view. With full-disk vector magnetograms, such a problem will be solved.

Flare kernels and ribbons were often found to be almost cospatial with the intersection of separatrices or QSLs with the solar surface [20-26]. Those authors related the ribbons to the reconnection area, which tends to be a magnetic null point or QSL. In their works, they generally picked up the flare ribbon images during the flare process. According to the reconnection model, flare ribbons should be located at the footpoints of the inner separatrix (or QSL). However, we stress that the inner separatrix (or QSL), which is directly linked to the reconnection area, might be difficult to derive with the current techniques of magnetic field extrapolation. The derived QSLs in this paper, and in some of the previous works, actually correspond to the outer separatrices (or QSLs), whose footpoints are cospatial with the final location of flare ribbons. If we compare the flare ribbon at any time with the derived QSLs, the two might always be roughly cospatial, since in most flares the moving distance of a flare ribbon is only $\sim 10^{\prime \prime}$ (e.g. [27]), which is of the order of the spatial resolution of many previous telescopes. With the unprecedented resolutions of both vector magnetograms and UV images by Solar Dynamics Observatory (SDO), more accurate comparisons between magnetic QSLs and the final locations of flare ribbons will be very meaningful.

The prediction of the flare occurrence is improving greatly. In this paper we propose a theoretical conjecture to predict the final locations of flare ribbons before the flare occurs. By assuming a suitable reconnection rate, we can even further predict the lifetime of a flare before it occurs, which will greatly enhance our capacity of space weather forecast.

The authors thank P. Démoulin for helpful suggestions. This work was supported by the National Natural Science Foundation of China (11025314, 10878002, and 10933003) and the National Basic Research Program of China (2011CB811402). PFC is also supported by an open research program of National Astronomical Observatories of China.

1 Pallavicini R. The role of magnetic loops in solar flares. Roy Soc London Phil Trans Ser A, 1991, 336: 389-399
2 Carmichael H. A Process for flares. NASA Spec Pub, 1964, 50: 451456

3 Sturrock P A. Model of the high-energy phase of solar flares. Nature, 1966, 211: 695-697

4 Hirayama T. Theoretical model of flares and prominences. I: Evaporating flare model. Solar Phys, 1974, 34: 323-338

5 Kopp R A, Pneuman G W. Magnetic reconnection in the corona and the loop prominence phenomenon. Solar Phys, 1976, 50: 85-98

6 Hudson H S. Global properties of solar flares. Space Sci Rev, 2011, 158: 5-41

7 Nolte J T, Gerassimenko M, Krieger A S, et al. Study of the post-flare loops on 29 July 1973. Solar Phys, 1979, 62: 123-132

8 Chen P F, Fang C, Tang Y H, et al. Simulation of magnetic reconnection with heat conduction. Astrophys J, 1999, 513: 516-523

9 Chen P F, Fang C, Ding M D, et al. Flaring loop motion and a unified model for solar flares. Astrophys J, 1999, 520: 853-858

10 Titov V S, Hornig G, Démoulin P. Theory of magnetic connectivity in the solar corona. J Geophys Res (Space Phys), 2002, 107: 1164-1176

11 Priest E R, Forbes T G. Magnetic flipping-Reconnection in three dimensions without null points. J Geophys Res, 1992, 97: 1521-1531

12 Handy B N, and 47 colleagues. The transition region and coronal explorer. Solar Phys, 1999, 187: 229-260

13 Ai G X. Solar magnetic field telescope. Pub Beijing Astron Observatory, 1987, 9: 27-36

14 Wiegelmann T. Optimization code with weighting function for the reconstruction of coronal magnetic fields. Solar Phys, 2004, 219: 87-108

15 Démoulin P. Extending the concept of separatrices to QSLs for magnetic reconnection. Adv Space Res, 2006, 37: 1269-1282

16 Wang H, Yan Y, Sakurai T, et al. Topology of magnetic field \& coronal heating in solar active regions. Solar Phys, 2000, 197: 263-273

17 Somov B V. New theoretical models of solar flares. Soviet Phys Uspekhi, 1985, 28: 271-272

18 Longcope D W, Kankelborg C C. Topology is destiny: Reconnection energetics in the corona. Earth Planet Space, 2001, 53: 571-576

19 Shibata K. Evidence of magnetic reconnection in solar flares and a unified model of flares. Astrophys Space Sci, 1999, 264: 129-144

20 Gorbachev V S, Somov B V. Photospheric vortex flows as a cause for two-ribbon flares-A topological model. Solar Phys, 1988, 117: 77-88

21 Mandrini C H, Démoulin P, Henoux J C, et al. Evidence for the interaction of large scale magnetic structures in solar flares. Astron Astrophys, 1991, 250: 541-547

22 Démoulin P, Mandrini C H, Rovira M G, et al. Interpretation of multiwavelength observations of November 5, 1980 solar flares by the magnetic topology of AR 2766. Solar Phys, 1994, 150: 221-243

23 van Driel-Gesztelyi L, Hofmann A, Démoulin P, et al. Relationship between electric currents, photospheric motions, chromospheric activity, and magnetic field topology. Solar Phys, 1994, 149: 309-330

24 Schmieder B, Aulanier G, Démoulin P, et al. Magnetic reconnection driven by emergence of sheared magnetic field. Astron Astrophys, 1997, 325: 1213-1225

25 Masson S, Pariat E, Aulanier G, et al. The nature of flare ribbons in coronal null-point topology. Astrophys J, 2009, 700: 559-578

26 Su Y, van Ballegooijen A, Schmieder B, et al. Flare energy build-up in a decaying active region near a coronal hole. Astrophys J, 2009, 704: $341-353$

27 Wang H, Qiu J, Jing J, et al. Study of ribbon separation of a flare associated with a quiescent filament eruption. Astrophys J, 2003, 593: $564-570$

Open Access This article is distributed under the terms of the Creative Commons Attribution License which permits any use, distribution, and reproduction in any medium, provided the original author(s) and source are credited. 